Gynäkologe 2017·50:838

https://doi.org/10.1007/s00129-017-4140-1

Online publiziert: 15. September 2017

๑) Springer Medizin Verlag GmbH 2017

CrossMark

\author{
W. Jonat ${ }^{1} \cdot$ W. Janni ${ }^{2}$ \\ ${ }^{1}$ Klinik für Gynäkologie und Geburtshilfe, Campus Kiel, Universitätsklinikum Schleswig-Holstein, Kiel, \\ Deutschland \\ ${ }^{2}$ Frauenklinik, Universitätsklinikum Ulm, Ulm, Deutschland
}

\title{
Krebs und Schwangerschaft
}

auch in Kontext einer Krebsbehandlungen zu erhalten und den Betroffenen so die Chance auf eine spätere Schwangerschaft zu geben - ein Wissen, das bereits ganz am Anfang der Behandlung wichtig ist.

Ein für Gynäkologinnen und Gynäkologen meist nur wenig verfügbares Wissen sind die besonderen Aspekte zu anästhesiologischen Verfahren während der Schwangerschaft, die Herr Fröba in seinem Beitrag vermittelt. Einem starken Wandel unterliegt der Umgang mit Zervixdysplasien und -karzinomen, insbesondere auch im Zusammenhang mit einer vorliegenden oder einer geplanten Schwangerschaft, wie Kollege Bauer in seinem Beitrag referiert. Bauer gibt in diesem Heft einen Überblick über die bunte, manchmal unübersichtliche Palette der gestationsbedingten Trophoblasttumoren.

Auch wenn Krebserkrankungen während und direkt nach der Schwangerschaft insgesamt selten sind, die Herausforderung ist eine besondere, und unsere Verantwortung für die bestmögliche Betreuung und Therapie im Sinne beider Leben ist besonders hoch. Wir hoffen, mit diesem Themenheft einen kleinen Beitrag zu leisten, der hohen Verantwortung gerecht $\mathrm{zu}$ werden, und wünschen Ihnen eine erkenntnisreiche Lektüre.

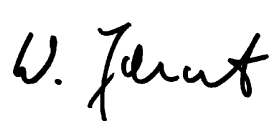

W. Jonat

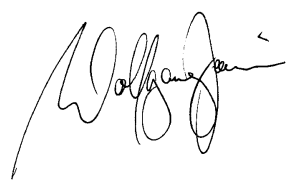

W. Janni

\section{Korrespondenzadresse}

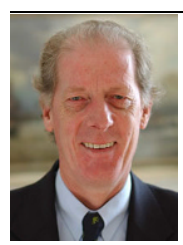

Prof. Dr. W. Jonat

Klinik für Gynäkologie und Geburtshilfe, Campus Kiel, Universitätsklinikum Schleswig-Holstein Arnold-Heller-Straße 3, 24105 Kiel, Deutschland Walter.Jonat@uksh.de

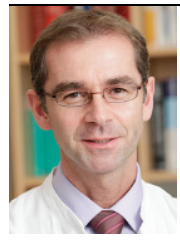

Prof. Dr. W. Janni

Frauenklinik, Universitätsklinikum Ulm

Prittwitzstr. 43, 89075 Ulm, Deutschland wolfgang.janni@uniklinikulm.de

Interessenkonflikt. W. Jonat und W. Janni geben an, dass kein Interessenkonflikt besteht. 